\title{
Fat shaming is making people sicker and heavier
}

\author{
Cite as: CMAJ 2019 June 10;191:E649. doi: 10.1503/cmaj.109-5758
}

Posted on cmajnews.com on May 23, 2019.

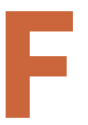

at shaming is harmful to health and may drive weight gain, said presenters at the Canadian Obesity Summit, recently held in Ottawa.

Anti-fat bias is rampant in all parts of society, including medicine, said Angela Alberga, an assistant professor in the department of health, kinesiology and applied physiology at Concordia University. More than three in five adults with obesity encounter weight bias from health professionals, according to Obesity Canada. Some medical ethicists even argue that this social pressure is justified to promote weight loss.

But the harms of fat shaming are well documented, Alberga said. Studies show that exposure to weight bias triggers physiological and behavioural changes linked to poor metabolic health and increased weight gain. "You actually experience a form of stress," Alberga explained. Cortisol spikes, self-control drops and the risk of binge eating increases, she said.

The more people are exposed to weight bias and discrimination, the more likely they are to gain weight and become obese, even if they were thin to begin with. They're also more likely to die from any cause, regardless of their body mass index (BMI).

Fat shaming is also linked to depression, anxiety, low self-esteem, eating disorders and exercise avoidance, Alberga said. There's emerging evidence that the severity of harm increases when people internalize weight bias and turn it on themselves. In one study, participants with high levels of internalization of weight-bias had three times greater odds of having metabolic syndrome than those with low levels, even after controlling for BMI and other risk factors. "It's a really complex relationship" that goes beyond energy-in-energy-out, Alberga said.

It's estimated that two in five Americans with a higher than "normal" BMI have internalized weight bias. But you don't have to be overweight or obese to suffer

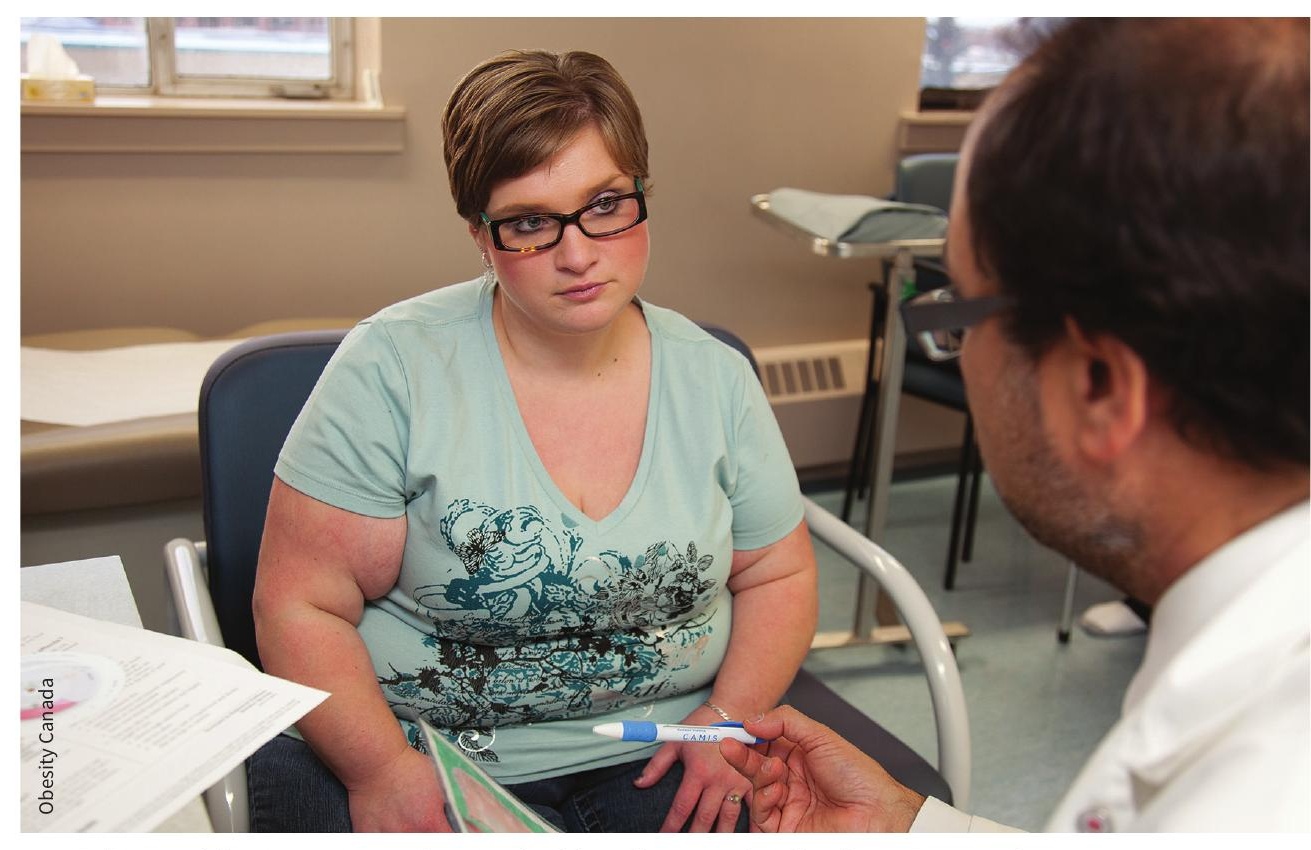

Weight bias and discrimination undermine health and may make it harder to lose weight.

harm, Alberga noted. "Weight-bias internalization can occur with any body size."

Better research is needed to understand the unique harms of internalizing anti-fat attitudes and assumptions, she said. Most studies have been cross-sectional and focused on samples of white women, and not much is known about interventions to reduce internalization of weight bias. Losing weight, for example, doesn't seem to make an impact.

According to Sara Kirk, a professor of health promotion at Dalhousie University and scientific director of the Healthy Populations Institute, tackling weight bias must start in health care. Judgmental responses of disgust, anger or blame from health professionals jeopardize care and discourage many patients from seeking help for health issues. "This is a systemic issue we need to be addressing," Kirk said.

At Dalhousie, Kirk's team created an artsbased workshop to teach trainees about the complexity of obesity, evoke empathy, and challenge stereotypes. A pilot demonstrated "significant reduction in anti-fat attitudes," Kirk said. About 1200 health care trainees have received the training to date. "Unfortunately, we still don't have long-term data on these students," Kirk said.

Beyond personal biases, the medical profession must also address bias in clinical guidelines. According to Toronto-based dietitian Maria Ricupero, many guidelines promote weight loss without acknowledging weight bias or environmental factors. Instead, "they emphasize what the individual can do to make change, which leads to guilt, shame and judgment." Ricupero argues the focus needs to shift from weight to health. "We know health benefits can be achieved when people are focusing on behaviours."

Canadian practice guidelines for obesity currently in development will aim to redress that imbalance, said Alberga. "We'll address weight bias and stigma in a dedicated chapter."

Lauren Vogel, CMAJ 